\title{
S-16-6 Neuronal Differentiation of Human Neuroblastoma Cells by Retinoid
}

\author{
T.Sugimoto, ${ }^{1}$ Y.HoriI, ${ }^{1}$ T.SAwada, ${ }^{1}$ S.Fushiki, ${ }^{2}$ \\ Y.SUZUKI, ${ }^{3}$ and O.TAGAYA ${ }^{3}$
}

\begin{abstract}
${ }^{1}$ Department of Pediatrics and ${ }^{2}$ Research Institute for Neurological Diseases and Geriatrics, Kyoto Prefectural University of Medicine, Kyoto 602 (Japan) and ${ }^{3}$ Eisai Co. Ltd., Bunkyo, Tokyo 112 (Japan)

I. INTRODUCTION

NB (Neuroblastoma), one of the most common malignant solid tumor in childhood, arises from primitive sympathetic neuroblasts. The prognosis of patients with advanced NB is still poor, despite recent progress in chemotherapy. Induction of differentiation of $N B$ in vitro by chemical and biological agents has been reported (1). In addition therapeutic trials for the induction of differentiation in vivo have been attempted. These trials, however, have been not proved successful. Recently a new synthetic polyprenoic acid, E5166, which has properties similar to RA (retinoic acid) and is less toxic than synthetic RA, have been described by Muto et al (2).

In this study two human NB cell lines, KP-N-RT and SK-N-DZ, were differentiated in vitro by E5166. Subsequently the effect of oral administration of E5166 on tumor growth in NB-bearing athymic mice was studied. The results of our studies suggest a possible therapeutic role for E5166 in vivo. Hopefully the differentiation of induction of NB may lead to the total eradication of residual NB cells after extensive chemotherapy and surgery.
\end{abstract}

II. MATERIALS AND METHODS

1. Human NB cell lines. Two human NB cell lines (KP-N-RT and SK-N-DZ) were cultured (2).

2. Morphological differentiation induction of NB cell lines by E5166 in liquid culture. E5166 (Eisai, Co. Ltd., Tokyo, Japan)(Fig. 1)(2) was dissolved in dimethyl sulfoxide (the final solvent dilution being $0.1 \%$ by volume). An optimal concentration of E5166, for the in vitro induction of morphological differentia tion $(1,3)$, was determined by adding this to cultures at concentrations ranging from 0.8 to $3.3 \times 10^{-5} \mathrm{M}$. Control cultures were established containing $0.1 \%$ solvent alone. Medium in the cultures was replaced every 4 days, and cells were maintained for a total of 12 days.

Fig. 1. Chemical Structure of E5166.

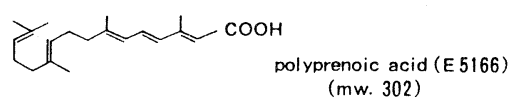

3. Indirect immunofluorescence of neurofilament. For the determination of neurofilament expression, adherent NB cells on coverslips were fixed with methanol washed and stained with appropriately titrated neurofilament monoclonal antibody (Bio-Science, Emmenbrucke, Switzerland) (3).

4. Assessment of morphological differentiation in liquid culture. Cells were plated into 60-mm Petri dishes containing $10 \times 10-m m-s i z e d$ coverslips. Coverslips were stained after E5166 treatment. Two hundred cells were examined by light 
microscopy and scored as morphologically differentiated, if they possessed one or more processes at least twice as long as the soma diameter (1).

5. N-myc and $\mathrm{c}-\mathrm{src}$ expressions and $\mathrm{N}$-myc ampification. N-myc and c-src expressions and $\mathrm{N}-m y c$ amplification were determined by Northern and Southern blot analyses (4). The following cloned DNA fragments were used as probes: $\mathrm{N}$-myc, a $1.0 \mathrm{~kb}$ EcoRI-BamHI fragment of pNb-1; v-src, a $0.8 \mathrm{~kb}$ PvuIIE fragment and a $0.255 \mathrm{~kb}$ PstI fragment of pTT107; $\beta$-actin, a $1.2 \mathrm{~kb}$ Pst fragment of pAL41 (4).

6. Effect of oral administration of E5166 on NB-bearing athymic mice. KP-N-RT NB cells $\left(10 \times 10^{6}\right.$ or $\left.40 \times 10^{6}\right)$ were subcutaneously inoculated into the dorsal area of athymic mice. E5166 (40 or $200 \mathrm{mg} / \mathrm{kg})$ in $0.1 \mathrm{ml}$ of peanut oil was orally administered by a stomach tube for a total of 4 weeks ( 5 days/week) either one day after tumor inoculation or after the formation of tumor (5-mm in diameter).

Peanut oil $(0.1 \mathrm{ml})$ alone was given to the control mice. Mice were examined at weekly intervals to detect tumor growth. Fourteen weeks after the inoculation of $\mathrm{NB}$ cells mice were sacrificed and the excised tumor wet weight was measured. Effect of E5166 on tumor cells was also histologically examined.

\section{RESULTS}

1. Morphological differentiation by E5166 in liquid culture. Ten days after beginning the experiment between 77 and $92 \%$ of the NB cells appeared morphologically differentiated. The dose of E5166, where optimal diffrentiation occured, was $1.7 \times 10^{-5} \mathrm{M}$. In the absence of $\mathrm{E} 5166$ a maximum of $35 \%$ of cells appeared differentiated. With regard to $S K-N-D Z$ cells, the maximum number of cells showing a differentiated morphology (45\%) was obtained by treated cells with $1.7 \times 10^{-5} \mathrm{M}$ E5166 for 10 days. Control cells showed $7 \%$ of the cells with a differentiated phenotype. Scanning electron microscopy showed the E5166 treated KP-N-RT cells aggregated and formed cellular clusters that were connected with long bundled neuronal networks after 12 day exposure to E5166. In contrast, control cells possessed spindle-shaped soma with short neurite possessed (Fig. 2). After the treatment of E5166 for 12 days, SK-N-DZ cells also showed long neurite processes.

2. Expression of neurofilament in E5166-treated NB cells. Three \% of non-treated KP-N-RT cells with short neurite processes were stained with neurofilament antibody, whereas most of neuronal bundled networks, induced by E5166 treatment for 12 days, were strongly stained.

3. $N$-myc and $c-s r c$ expressions and N-myc Amplification during neuronal differentiation. A detectable decrease in the levels of $\mathrm{N}$-myc mRNA was observed by 24 hrs after treatment of KP-N-RT cells with E5166. Five days after treament, there was a $75 \%$ decrease in the levels of $\mathrm{N}-\mathrm{myc}$ expression in E5166-treated KP-N -RT cells, as compared with control cells. The expression remained at this level during the 10 day exposure to E5166. Similar decreased in N-myc expression (75\%) were observed in E5166-treated SK-N-DZ cells after 10 days exposure. In contrast, no differences in $\mathrm{N}-\mathrm{myc}$ amplification was shown between E5166treated and control KP-N-RT and SK-N-DZ cell lines (Fig. 3).

There was an increase $(300-400 \%)$ in the level of c-src expression (4.0 kb) in neuronally differentiated $K P-N-R T$ and $S K-N-D Z N B$ cell lines using the PVuIIE fragment (Fig. 3). Hybridization with the PstI fragment (upstream of the PvulIE fragment) revealed the same $4.0 \mathrm{~kb}$ band and the same results (Data not shown).

4. Effect of oral administration of E5166 on NB-bearing athymic mice. When tumor developed to $5-\mathrm{mm}$ in diameter, mice were treated with either 40 or $200 \mathrm{mg} /$ $\mathrm{kg}$ of E5166. No significant difference was noted in the inhibition of tumor growth between control and E5166-treated mice. Whereas in the mice treated with E5166 (200mg/kg) one day after the tumor inoculation $\left(40 \times 10^{6}\right)$, tumor wet weight was significantly inhibited as compared with control mice $(p<0.05)$ (Fig. 4$)$. 
Histological observations of E5166-treated tumors revealed the reduced number of mitotic cells, the appearance of ganglion-like cells and the well-developed stromal tissues, suggesting the neuronal differentiation of NB cells (Fig. 5).

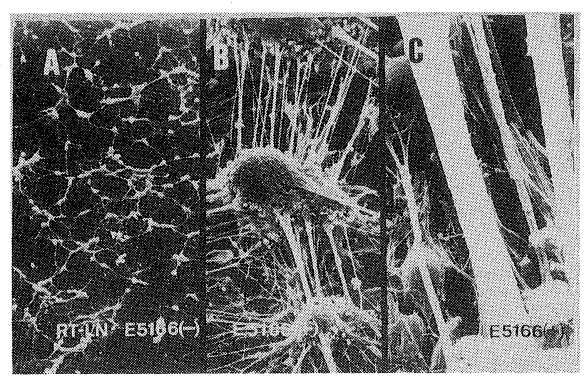

Fig. 2. Scanning electron microscopy of control (A) and E5166-treated differentiated KP-N-RT cells $(B, C)$.

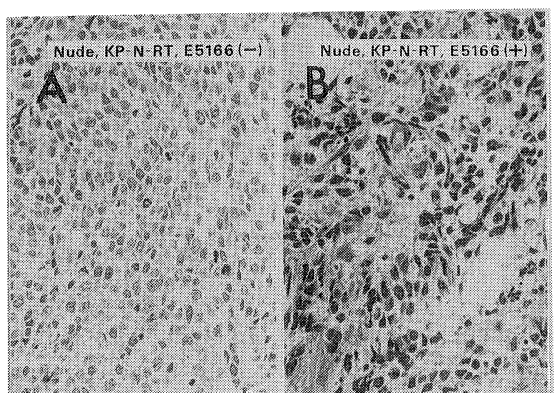

Fig. 4. Effect of oral administration of E5166 on tumor weight in NB-bearing mice. Mice were treated with E5166 for 4 weeks one day after tumor inoculation $\left(40 \times 10^{6}\right)$.

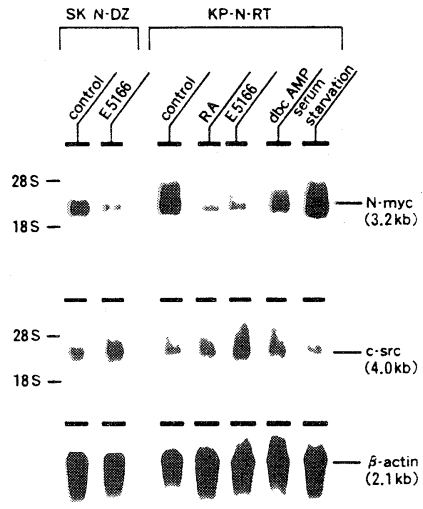

Fig. 3. Down-regulation of $N-m y c$ and up-regulation of c-src expressions during neuronal differentiation.

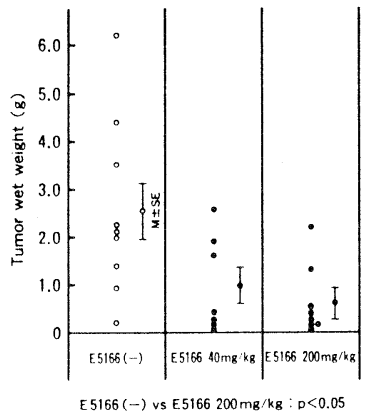

Fig. 5. Histological effect of E5166 on NB cells in athymic mice. A: Control, B: E5166-treated.

\section{DISCUSSION}

A new synthetic polyprenoic acid, E5166, is a dervatives of RA that has a high binding affinity to cellular retinoic acid-binding protein (2).

In the present study, two human NB cell lines, KP-N-RT and SK-N-DZ, were induced to differentiate in liquid culture. Scanning electron microscopy showed 
that E5166-treated KP-N-RT cells aggregated, forming cellular clusters that were conencted with long bundled neurite processes, indicating neuronal differentiati on (Fig. 2). Transmission electron microscopy indicated the development of Golgi apparatus, mitochondria and neural tubules, analogous to those found in differentiated cells. However no significant increases in dense core (catecholamine) granules was observed in E5166-treated cells (Data not shown). Nerofi1ament is an intermediate filament of neurons and wel1-differentiated neuron express this protein (5). The neurofilament antibody reacted with E5166-treated KP-N-RT cells, indicating neuronal differentiation of this cell 1 ine by E5166 (Data not shown).

The formation of NB colonies in soft agar was inhibited by increasing concentration of E5166 using KP-N-RT and SK-N-DZ cell lines. Following treatment with E5166, neurite cells with processes forming differentited colonies were also observed (Data not shown).

Although the mechanism of E5166 in NB differentiation is not known, decreased $\mathrm{N}$-myc expression was observed in E5166-induced neuronal NB cells (Fig. 3 ), like that of RA-induced neuronal NB cells (6). This down-regulation of $\mathrm{N}-\mathrm{myc}$ expression was also an early event in the differentiation process. These findings appear to be related to the differentiation process and not to decreased cell growth, since serum stavation did not affect the level of $\mathrm{N}$-myc expression. our results, indicating the down-regulation of $\mathrm{N}$-myc expression during neuronal differentiation in vitro, support previous observations concerning the relationship between $\mathrm{N}$-myc oncogene and NB differentiation ( 6 ). With regard to the c-src oncogene, the expression increased in neuronally differentiated-NB cells (Fig. 3). Our results correspond well with previous reports elucidating the relationship between $c-s r c$ expression and neuronal differentiation ( 7 ).

In order to assess the potential clinical application and the limitation of E5166 treatment in patients with $\mathrm{NB}$, the experiment of athymic mice was conducted. In mice whose tumors developed to $5-\mathrm{mm}$ in diameter, no significant inhibition of tumor growth was observed between control and E5166-treated mice. However tumor weight, treated one day after tumor inoculation with higher dose of E5166 $(200 \mathrm{mg} / \mathrm{kg})$, was significantly inhibited (Fig. 4) and morphological differentiation was observed in excised tumors (Fig. 5), indicating differentiation therapy could be effective in the case of occult tumors.

E5166 is a potent reagent to induce neuronal differentiation in vitro and in vivo. This has equivalent differentiation activity to similar concentration of a 11-trans RA, can be administered to patients orally, and is 5 to 10 times less toxic than al1-trans RA (1). The clinical usefulness of E5166 in NB is suggested by the neuronal differentiation and the inhibition of tumor growth in both in vitro and in vivo systems. The drug may be useful in advanced NB patients to differentiate and eradicate residual tumor cells after surgery and chemotherapy.

\section{REFERENCES}

(1) Sugimoto, T. Sawada, T., Matsumura, T. et al (1987): Cancer Res., 47, 54335438 .

(2) Muto, Y. and Moriwaki, H. (1984): J. Nat1. Cancer Inst., 73, 1389-1393.

(3) Sugimoto, T., Sawada, T. Matsumura, T. et a 1 (1988): in Adv. Neuroblastoma Res., ed. by Evans, A. E., Alan R. Liss, New York, pp337-351.

(4) Hori, Y., Sugimoto, T., Sawada, T. et al (1989): Int. J. Cancer, 43, 305309.

(5) Shaw, G. and Weber, K. (1982): Nature (Lond.), 298, 277-279.

(6) Thiele, C.J., Reynolds, C.P. and Israel, M. A. (1985): Nature (Lond.), 313, 404-406.

(7) Brugee, J.S., Cotton, P.C., Queral, A.E. et al (1985): Nature (Lond.), 316, $554-557$. 